\title{
Gamma Knife Radiosurgery for Mesial Temporal Lobe Epilepsy
}

\author{
John D. Rolston, ${ }^{1}$ Mark Quigg, ${ }^{2}$ and Nicholas M. Barbaro ${ }^{1,3}$ \\ ${ }^{1}$ Department of Neurological Surgery, University of California at San Francisco, San Francisco, \\ CA 94143, USA \\ ${ }^{2}$ Department of Neurology, University of Virginia, Charlottesville, VA 22908, USA \\ ${ }^{3}$ Department of Neurology, University of California at San Francisco, San Francisco, CA 94143, USA
}

Correspondence should be addressed to Nicholas M. Barbaro, barbaron@neurosurg.ucsf.edu

Received 30 June 2011; Revised 30 August 2011; Accepted 6 September 2011

Academic Editor: Giangennaro Coppola

Copyright (C) 2011 John D. Rolston et al. This is an open access article distributed under the Creative Commons Attribution License, which permits unrestricted use, distribution, and reproduction in any medium, provided the original work is properly cited.

\begin{abstract}
Many patients with mesial temporal lobe epilepsy continue to have seizures despite medical therapy. For these patients, one recourse is surgical resection of the mesial temporal lobe, with its attendant risks. Noninvasive treatment with Gamma Knife radiosurgery is under active investigation as a possible alternative to open surgery. Accumulated evidence from multiple studies shows radiosurgery to be comparable in outcomes to surgical resection. A definitive randomized, controlled trial, the Radiosurgery or Open Surgery for Epilepsy (ROSE) trial, is currently underway, and further investigation of this promising treatment is crucial in our advancement of alternative therapies to treat refractory epilepsy.
\end{abstract}

\section{Introduction}

Mesial temporal lobe epilepsy (MTLE) is the most common etiology of medically intractable epilepsy in adults $[1,2]$. The standard of treatment for medically intractable MTLE is anterior temporal lobectomy (ATL), definitively demonstrated as superior to best medical care in a randomized, prospective trial [3]. ATL, however, is not perfect and has risks shared with any open procedure, including anesthesia complications, infection, neurological deficits, and bleeding $[4,5]$. Moreover, despite the proven utility of ATL [3], the procedure remains underused [6]. This lack of referral is generally due to reticence on behalf of the referring neurologist, but the origin of this reticence has yet to be completely elucidated [7].

\section{Technology}

Noninvasive methods of intracranial surgery, therefore, have been sought to address these potential risks and increase the pool of potential surgical candidates. Radiosurgery (RS) with the Leksell Gamma Knife (Elekta AB, Stockholm, Sweden) has been used in over half a million patients with neurological disorders, providing a robust, noninvasive means of treating intracranial pathology. Fundamental to RS is ionizing radiation, that is, radiation capable of stripping electrons from atoms or molecules, resulting in the breakage of chemical bonds, the creation of new bonds, or the production of free radicals $[8,9]$. In the case of RS of the brain, ionizing radiation damages cellular DNA. Damage to DNA, if it not repaired, can lead to apoptosis, necrosis, or potentially carcinogenesis [9]. Cells have differential susceptibility to the effects of ionizing radiation, typically attributed to their degree of DNA synthesis. For example, actively proliferating tissue, like vasculature, is more sensitive than terminally differentiated cells, like neurons. This ability to induce necrosis and apoptosis in tissue with ionizing radiation, and the ability of this radiation to penetrate tissue, has led to the use of radiation to destroy tumors and ablate tissue nonoperatively in targets deep within the skull, as first described by the Swedish neurosurgeon Leksell in 1951 [10].

Several sources of ionizing radiation are in clinical use. A proton beam accelerator strips protons of their electrons and aims them at a target. The mass of protons is helpful in that their mass makes them less likely to scatter when entering tissue. Furthermore, they tend to distribute their most intense radiation in a concentrated region toward the end 
of their path, a phenomenon known as the Bragg peak [9]. Despite these advantages, the limited availability of proton beam sources and their high cost limits widespread use [11].

The other carriers of ionizing radiation, photons, are easier to generate. The tradeoff is that photons, being massless, tend to scatter more readily than protons and are therefore more complicated to aim $[8,9,11]$. Rather than the aiming of a single proton beam, photons are best aimed by concentrating multiple weak sources to a single intense focal point. Therefore, the intersection of the beams receives far more radiation than the tissue in the path of any one beam by itself. This reduces side-effects of radiation in normal tissue and concentrates the radiation dose in the targeted tissue.

The two main sources of ionizing photons are linear accelerators, like those produced by the CyberKnife (Accuray, Inc.; Sunnyvale, Calif, USA), and radioactive elements like cobalt-60, as used in the Gamma Knife [11]. Linear accelerators use a single source that is steered about to deliver photon beams to a common target from different directions. Conversely, in the Gamma Knife, 200 separate cobalt-60 sources are housed inside multiple chambers and focused to a single target. The target, in turn, is determined and maintained with the patient installed into a stereotactic headframe.

\section{Efficacy in Seizure Remission}

Gamma Knife RS has been used to treat epilepsy stemming from a variety of etiologies, including supratentorial tumors, hamartomas, cavernous malformations, and arteriovenous malformations. The data concerning these diseases are reviewed elsewhere [12-14]. Mesial temporal lobe epilepsy is the subject of the remainder of this review.

The reported trials of RS for well-defined MTLE show a wide variation of clinical efficacies (Table 1); therefore, a detailed examination of differences in protocol and techniques is required. In general, higher doses directed to anatomical targets removed in standard open surgery for MTLE have proven more effective than lower doses or more restrictive targets. In addition, dose and targeting have been difficult to extrapolate from animal experiments, and targeting protocols remain largely empirically derived.

The first reported use of RS for MTLE was by Régis et al. in 1995 in the treatment of a 25-year-old patient with longstanding complex partial seizures [24]. After Gamma Knife treatment, with the use of $25 \mathrm{~Gy}$ for a $6.44 \mathrm{~mL}$ $50 \%$ isodose volume of tissue in the right amygdala and hippocampus, the patient was seizure-free for 16 months of followup, though still taking antiepileptic drugs. The case encouraged Régis et al. to attempt a larger trial of 7 refractory MTLE patients, the results of which were reported in 1999 [15] with a dose of $25 \mathrm{~Gy}$ delivered to a mean $50 \%$ isodose volume of $6.5 \mathrm{~mL}$. The target included the entorhinal cortex, head of the hippocampus, anterior hippocampal body, and the efferent amygdala, regions found to be important in seizure remission [25]. Overall, 6 of the 7 patients were seizure-free (Engel class IA) at followup (average 34 months, range 22-61 months). The only noted side-effects were headaches, which resolved, and a homonymous superior quadrantanopia in a single patient, as often occurs with standard ATL $[4,5,15]$.

A variety of single-center case reports and case series followed the promising results of Régis [16-18, 20, 21]. With one exception, these series used doses smaller than $25 \mathrm{~Gy}$, and rates of seizure remission were low. The exception is the study by Hoggard et al. reported in 2008 [21]. Notably, targeting did not differ significantly from early data from Régis et al. [15]. A potential discrepancy is the role of the number of "shots," individual dose isocenters that when strung together allow tailoring of the focus point into complex, anatomically conformal shapes. Régis's early studies restricted the number of shots to 2, as used by Hoggard. Later patients in Régis' practice were treated with $>2$ isodose centers. It remains unclear, however, if targeting protocols could account for divergent results with similar RS doses.

During this same period, Régis et al. concluded the first prospective (nonrandomized), multicenter study of RS for MTLE in Europe, this time using a dose of 24-25 Gy delivered to a brain volume of 5.5-9 mL [19]. In this trial, 13 of 21 patients $(62 \%)$ were seizure-free at 2 years. Five patients experienced headache, nausea, vomiting, imbalance, and depression, and 9 experienced visual field deficits. Neuropsychological testing using the Wechsler Memory Scale found no statistically significant changes after Gamma Knife treatment as compared to baseline [19].

Following the prospective trial by Régis et al. [19], Barbaro et al. reported a prospective, multicenter phase 3 trial of RS for MTLE in 30 patients [22]. With dose response unclear at the time of trial creation, patients were randomized to a high ( $24 \mathrm{~Gy}, n=13)$ or low dose (20 Gy, $n=$ 17) of radiation delivered to the hippocampal head, anterior hippocampal body, anterior parahippocampus, entorhinal cortex adjacent to the collateral sulcus, and the efferent amygdala [22]. 50\% isodose volumes were restricted to 5.5$7.0 \mathrm{~mL}$ attained with 2-6 "shots." Ten patients in each group were seizure-free at 24 months of followup, resulting in a remission rate of $77 \%$ in the high-dose group, and $59 \%$ in the low-dose group.

At the same time of the report by Barbaro et al., Vojtěch et al. [23] released a retrospective, long-term followup analysis of 14 patients receiving RS for MTLE, with markedly different results [23]. Initial doses were identical to that reported by Régis et al. [19], and, in fact, 6 of the patients were enrolled and reported in the European Multicenter trial [19]. Doses decreased with accumulated experience; of the 14 patients in the report, the last 6 were treated with $18 \mathrm{~Gy}$ because of clinically significant "prominent radiosurgical responses" associated with 25 Gy called for in the European Multicenter Protocol. None of the patients were seizurefree after 39-months [23]. Beyond dose-response effects, the authors discussed that other differences may have accounted for the poor response to RS, including differences in followup duration, classification of events, patient selection, and presurgical localization. Intriguingly, 7 of the patients then underwent open surgical resection (amygdalohippocampectomy), resulting in seizure freedom in 4 of these 7 operated patients $(57 \%)$ [23]. The authors note that eventual success 
TABLE 1: Trials of Gamma Knife radiosurgery for MTLE.

\begin{tabular}{|c|c|c|c|c|c|}
\hline Study & NO. of subjects & Dose (Gy) & Volume of treatment $(\mathrm{mL})$ & Minimum followup & Seizure freedom $^{\dagger}$ \\
\hline Régis et al. [15] & 7 & 25 & $6.25-6.9$ & $22^{*}$ & $6(86 \%)$ \\
\hline Cmelak et al. [16] & 1 & 15 & $\mathrm{n} / \mathrm{a}$ & 12 & 0 \\
\hline Kawai et al. [17] & 2 & 18 & $6.2-8.7$ & 18 & 0 \\
\hline Srikijvilaikul et al. [18] & 5 & 20 & $6.1-8.7$ & 24 & 0 \\
\hline Régis et al. [19] & 21 & 24 & $5.5-9$ & 24 & $13(62 \%)$ \\
\hline Prayson and Yoder [20] & 4 & 20 & $6.1-8.7$ & 24 & 0 \\
\hline Hoggard et al. [21] & 8 & 25 & $6.2 \pm 0.7$ & 24 & $3(37 \%)$ \\
\hline Barbaro et al. [22] & & & $5.5-7.5$ & 24 & \\
\hline High dose (24 Gy) & 13 & 24 & - & - & $10(77 \%)$ \\
\hline Low dose (20 Gy) & 17 & 20 & - & - & $10(59 \%)$ \\
\hline Vojtěch et al. [23] & & & $5.2-8.9$ & 39 & 0 \\
\hline High dose (25 Gy) & 6 & 25 & - & - & 0 \\
\hline Low dose (18-20 Gy) & 8 & $18-20$ & - & - & 0 \\
\hline Total & 92 & & & & $42(46 \%)$ \\
\hline High dose ( $\geq 24$ Gy) & 55 & & & & $32(58 \%)$ \\
\hline Low dose $(<24 \mathrm{~Gy})$ & 37 & & & & $10(27 \%)$ \\
\hline
\end{tabular}

${ }^{*}$ The manuscript lists both 22 and 24 months as the minimum followup [15]. Due to the discrepancy, we list the lower of the two values in this table.

${ }^{\dagger}$ Seizure freedom is defined as Engel Class IA.

with open surgery implied that the epileptic zone was correctly identified. Another interpretation not advanced by the authers but speculated in a report of the long-term followup of patients treated by the Régis group is that failed seizure remission in the latter report was associated with extension of the epileptic zone beyond traditional mesial structures [26].

The results of RS for MTLE, therefore, suggest that there is a narrow therapeutic window in dose response. In addition, patient selection with rigorous definition of the traditional epileptic target-unilateral mesial-may be critical to success with the "superselective" and restricted anatomic disruption offered by the GK technique.

\section{Timing of RS Effects}

The postoperative course of RS for MTLE appears similar to that of other types of RS. Significant radiographic changes become apparent between 9 and 12 months after surgery $[15,19,24,27,28]$. The typical radiographic pattern reveals T2 hyperintensity, presumably reflecting vasogenic edema, which begins at 9 months, peaking at 12, and resolving by 24-27 months [28]. Contrast enhancement (with gadolinium) follows a similar time course, but precedes the T2 changes, is typically ring-enhancing, and subsides quickly at 9-12 months after RS [28]. The mass effect induced by RS has a time course correlated to that of the T2 hyperintensity changes. After the hyperintensity and mass effect resolve, the remaining temporal lobe appears slightly atrophic. These changes are dose dependent; higher doses cause greater effects [28].

Magnetic resonance spectroscopy reveals that baseline levels of choline, creatine, and $\mathrm{N}$-acetylaspartate (NAA) were normal or slightly reduced before RS. At one year, a lactate peak appears, and choline, creatine, and NAA peaks are largely absent $[23,28]$. Also, there may be an increase in NAA in the contralateral amygdala and hippocampus following RS [23].

Clinical changes accompany those of neuroimaging that demonstrate development of the RS lesion. During the time following irradiation, there is often a significant increase in the frequency of auras (simple partial seizures), followed by the eventual decrease in seizure frequency $[19,22,23]$. The transient exacerbation and decline in auras (and sometimes in complex partial seizures) occurs earlier in those with higher doses [23]. New onset headaches occur within this early period and can precede frank neuroimaging changes [22]. The decline in seizures, when it occurs, tends to happen 12-18 months after radiation $[19,22]$, but also appears to correlate with dose; that is, higher doses tend to produce seizure freedom more rapidly than lower doses [22].

Timing and severity of neuroimaging changes may predict subsequent seizure remission. For example, the volume of contrast enhancement and T2 hyperintensity seen on MRIs obtained 12 months after RS correlated strongly with outcome. No patients who had T2-weighted volumes of edema $<200 \mathrm{~mL}$ at 12 months went on to experience seizure remission between 24 and 36 months [28]. Although the predictive value is not as great, the transient increase in auras correlates with the volume of T2-weighted edema, a clinical finding that could be useful in warning physicians to acquire a postoperative MRI [29].

\section{Safety}

Side effects of RS for MTLE have been reported by multiple groups and usually include headache, nausea, depression, visual field deficits, and, infrequently, more serious adverse events $[19,20,22,27]$. In the prospective studies of Régis et al. [19] and Barbaro et al. [22], incidence of new onset 
headaches was $14 \%$ and $70 \%$, respectively, and visual field deficits were $43 \%$ and $50 \%$, respectively.

Serious adverse events were reported by Barbaro et al. [22], Srikijvilaikul et al. [18], and Prayson and Yoder [20]. In the prospective trial of Barbaro et al., one patient in the highdose (24 Gy) group showed signs of increased intracranial pressure (papilledema, enlarging blinds spots, and new onset headaches) in a steroid-dependent fashion. This patient was treated with ATL, leading to remission of symptoms and seizures [22]. In the small study of 4 patients receiving RS by Prayson and Yoder, one patient died two weeks after receiving therapy, reportedly to "persistent seizure complications" [20]. Since 14 days is too short for development of the RS lesion, it is not clear if treatment was related to the patient's demise. This opinion is supported by autopsy that revealed mesial temporal sclerosis, but no vascular sclerosis, necrosis, perivascular inflammation, or microglial proliferation. These changes are present in the resected temporal lobes of 3 other patients who received RS for MTLE, but present only after a minimum of 18 months [20]. In the study of 5 patients by Srikijvilaikul et al., 2 of the patients died during the latent period following irradiation, one at 1 month and one at 1 year postoperatively [18]. As in the Srikijvilaikul et al. study, these were attributed to the complications of persistent seizures [18] and may best be summarized as sudden unexpected death in epilepsy (SUDEP).

Neuropsychological testing has also been used to detect more nuanced deficits following RS. As noted previously, Régis et al. noted no observable deficits in their prospective trial using the Wechsler Memory Scale [19]. Barbaro et al., in their prospective study, however, found verbal memory impairment in 15\% of patients using the Wechsler Memory Scale and the California Verbal Learning Test [22]. On the other hand, three patients (13\%) experienced improvements in verbal memory [22]. More detailed analysis of these patients at 24 months postirradiation, using the Boston Naming Test, California Verbal Learning Test, Wechsler Memory Scale, Trail Making Test, and Beck Depression Inventory, revealed no significant neuropsychological changes in the patients between their preoperative baseline and postirradiated states [30]. Importantly, quality of life scores was significantly improved, especially in those patients attaining seizure freedom [30]. Compared to patients undergoing surgical resection of the dominant hippocampus, wherein $60 \%$ of patients show impairment of verbal memory, the results of RS appear to be highly favorable [31].

The side effects and complications of RS for MTLE should be placed in context with those of standard open surgery. Both RS and ATL result in visual field defects. Mortality has occurred after ATL [4] and during the period of development of the radiation lesion after RS (albeit with mortalities occurring after less effective "low-dose" treatment protocols). ATL has caused transient (4\%) or permanent hemiparesis (2\%) [4]. Infections occur in $0.8 \%$ $[4,5]$, and bleeding in the immediate postoperative period requiring transfusion in $2.3 \%$ [5]. In comparison, neither of the large prospective RS trials reported infections (which could presumably occur by way of the stereotactic pins) $[19,22]$.

\section{Conclusions}

Multiple independent studies have confirmed the efficacy and safety of Gamma Knife RS as a treatment for refractory MTLE. Across 9 studies, $46 \%$ of patients achieved complete seizure freedom within 2 years or irradiation, and often earlier. When only those patients who received high-dose radiation ( $\geq 24 \mathrm{~Gy}$ ) are examined, seizure freedom occurs in $58 \%$ of patients (Table 1). Side effects are comparable to resective amygdalohippocampectomy, with neuropsychological outcomes possibly superior in those patients treated with the Gamma Knife. These findings across studies should be confirmed by the currently recruiting randomized clinical trial that compares RS to standardized ATL, the ROSE trial (http://ClinicalTrials.gov/ identifier NCT00860145). Due to the substantial morbidity of MTLE patients who are refractory to antiepileptic drugs, and due to the morbidity associated with invasive open surgery, our continued investigation of noninvasive radiosurgical treatments for epilepsy is critical.

\section{References}

[1] S. Wiebe, "Epidemiology of temporal lobe epilepsy," Canadian Journal of Neurological Sciences, vol. 27, supplement 1, pp. S6S10, 2000.

[2] P. Kwan and M. J. Brodie, "Early identification of refractory epilepsy," The New England Journal of Medicine, vol. 342, no. 5, pp. 314-319, 2000.

[3] S. Wiebe, W. T. Blume, J. P. Girvin, and M. Eliasziw, "A randomized, controlled trial of surgery for temporal-lobe epilepsy," The New England Journal of Medicine, vol. 345, no. 5, pp. 311-318, 2001.

[4] J. Engel, Surgical Treatment of the Epilepsies, Raven Press, 1993.

[5] S. McClelland III, H. Guo, and K. S. Okuyemi, "Populationbased analysis of morbidity and mortality following surgery for intractable temporal lobe epilepsy in the United States," Archives of Neurology, vol. 68, no. 6, pp. 725-729, 2011.

[6] P. de Flon, E. Kumlien, C. Reuterwall, and P. Mattsson, "Empirical evidence of underutilization of referrals for epilepsy surgery evaluation," European Journal of Neurology, vol. 17, no. 4, pp. 619-625, 2010.

[7] E. Kumlien and P. Mattsson, "Attitudes towards epilepsy surgery: a nationwide survey among Swedish neurologists," Seizure, vol. 19, no. 4, pp. 253-255, 2010.

[8] F. M. Khan, The Physics of Radiation Therapy, Lippincott Williams \& Wilkins, 2010.

[9] B. G. Haffty and L. D. Wilson, Handbook of Radiation Oncology: Basic Principles and Clinical Protocols, Jones and Bartlett, 2009.

[10] L. Leksell, "The stereotaxic method and radiosurgery of the brain," Acta chirurgica Scandinavica, vol. 102, no. 4, pp. 316319, 1951.

[11] M. P. Mehta, "The physical, biologic, and clinical basis of radiosurgery," Current Problems in Cancer, vol. 19, no. 5, pp. 265-329, 1995.

[12] M. Quigg and N. M. Barbaro, "Stereotactic radiosurgery for treatment of epilepsy," Archives of Neurology, vol. 65, no. 2, pp. 177-183, 2008.

[13] S. Batra, D. Lin, P. F. Recinos, J. Zhang, and D. Rigamonti, "Cavernous malformations: natural history, diagnosis and treatment," Nature Reviews Neurology, vol. 5, no. 12, pp. 659670, 2009. 
[14] J. L. Frazier, C. R. Goodwin, E. S. Ahn, and G. I. Jallo, "A review on the management of epilepsy associated with hypothalamic hamartomas," Child's Nervous System, vol. 25, no. 4, pp. 423 432, 2009.

[15] J. Régis, F. Bartolomei, M. Rey et al., "Gamma knife surgery for mesial temporal lobe epilepsy," Epilepsia, vol. 40, no. 11, pp. 1551-1556, 1999.

[16] A. J. Cmelak, B. Abou-Khalil, P. E. Konrad, D. Duggan, and R. J. Maciunas, "Low-dose stereotactic radiosurgery is inadequate for medically intractable mesial temporal lobe epilepsy: a case report," Seizure, vol. 10, no. 6, pp. 442-446, 2001.

[17] K. Kawai, I. Suzuki, H. Kurita, M. Shin, N. Arai, and T. Kirino, "Failure of low-dose radiosurgery to control temporal lobe epilepsy," Journal of Neurosurgery, vol. 95, no. 5, pp. 883-887, 2001.

[18] T. Srikijvilaikul, I. Najm, N. Foldvary-Schaefer et al., "Failure of gamma knife radiosurgery for mesial temporal lobe epilepsy: report of five cases," Neurosurgery, vol. 54, no. 6, pp. 1395-1404, 2004.

[19] J. Régis, M. Rey, F. Bartolomei et al., "Gamma knife surgery in mesial temporal lobe epilepsy: a prospective multicenter study," Epilepsia, vol. 45, no. 5, pp. 504-515, 2004.

[20] R. A. Prayson and B. J. Yoder, "Clinicopathologic findings in mesial temporal sclerosis treated with gamma knife radiotherapy," Annals of Diagnostic Pathology, vol. 11, no. 1, pp. 22-26, 2007.

[21] N. Hoggard, I. D. Wilkinson, P. D. Griffiths, P. Vaughan, A. A. Kemeny, and J. G. Rowe, "The clinical course after stereotactic radiosurgical amygdalohippocampectomy with neuroradiological correlates," Neurosurgery, vol. 62, no. 2, pp. 336344, 2008.

[22] N. M. Barbaro, M. Quigg, D. K. Broshek et al., "A multicenter, prospective pilot study of gamma knife radiosurgery for mesial temporal lobe epilepsy: seizure response, adverse events, and verbal memory," Annals of Neurology, vol. 65, no. 2, pp. 167175, 2009.

[23] Z. Vojtěch, V. Vladyka, M. Kalina et al., "The use of radiosurgery for the treatment of mesial temporal lobe epilepsy and long-term results," Epilepsia, vol. 50, no. 9, pp. 2061-2071, 2009.

[24] J. Regis, J. C. Peragut, M. Rey et al., "First selective amygdalohippocampal radiosurgery for 'mesial temporal lobe epilepsy', Stereotactic and Functional Neurosurgery, vol. 64, supplement 1, pp. 193-201, 1995.

[25] A. M. Siegel, H. G. Wieser, W. Wichmann, and G. M. Yasargil, "Relationships between MR-imaged total amount of tissue removed, resection scores of specific mediobasal limbic subcompartments and clinical outcome following selective amygdalohippocampectomy," Epilepsy Research, vol. 6, no. 1, pp. 56-65, 1990.

[26] S. Rheims, C. Fischer, P. Ryvlin et al., "Long-term outcome of gamma-knife surgery in temporal lobe epilepsy," Epilepsy Research, vol. 80, no. 1, pp. 23-29, 2008.

[27] J. Regis, F. Semah, R. N. Bryan et al., "Early and delayed MR and PET changes after selective temporomesial radiosurgery in mesial temporal lobe epilepsy," American Journal of Neuroradiology, vol. 20, no. 2, pp. 213-216, 1999.

[28] E. F. Chang, M. Quigg, M. C. Oh et al., "Predictors of efficacy after stereotactic radiosurgery for medial temporal lobe epilepsy," Neurology, vol. 74, no. 2, pp. 165-172, 2010.

[29] M. Quigg et al., "Localization of epileptic auras change after radiosurgery for mesial temporal lobe epilepsy," in Proceedings of the 65th Annual Meeting of the American Epilepsy Society, 2001.
[30] M. Quigg, D. K. Broshek, N. M. Barbaro et al., "Neuropsychological outcomes after Gamma Knife radiosurgery for mesial temporal lobe epilepsy: a prospective multicenter study," Epilepsia, vol. 52, no. 5, pp. 909-916, 2011.

[31] E. Stroup, J. Langfitt, M. Berg, M. McDermott, W. Pilcher, and P. Como, "Predicting verbal memory decline following anterior temporal lobectomy (ATL)," Neurology, vol. 60, no. 8, pp. 1266-1273, 2003. 


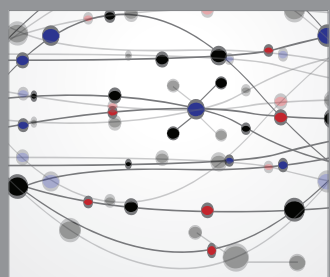

The Scientific World Journal
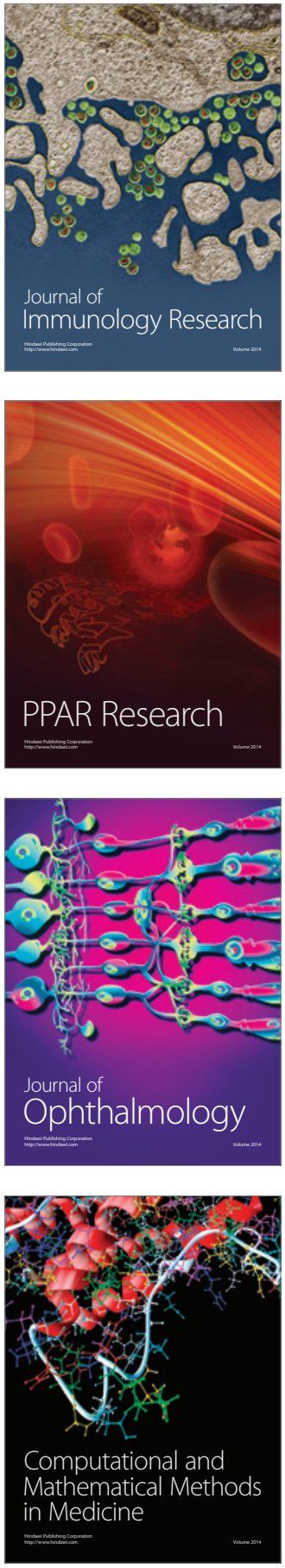

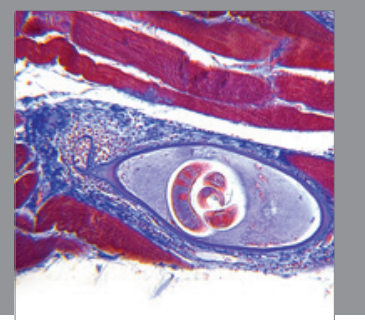

Gastroenterology

Research and Practice
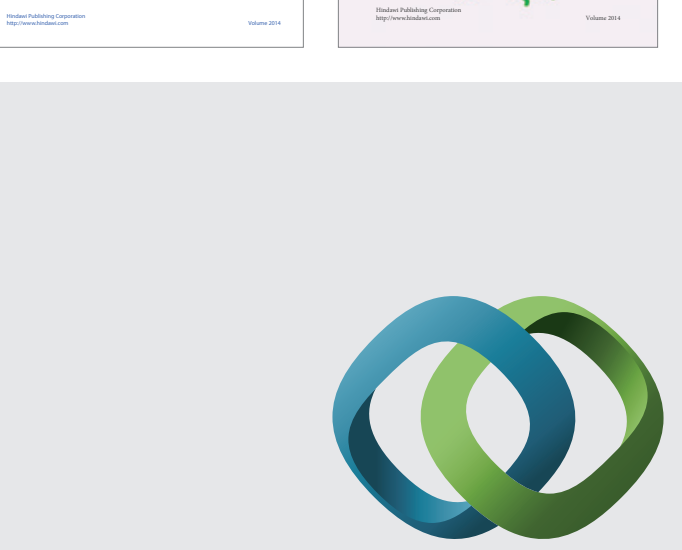

\section{Hindawi}

Submit your manuscripts at

http://www.hindawi.com
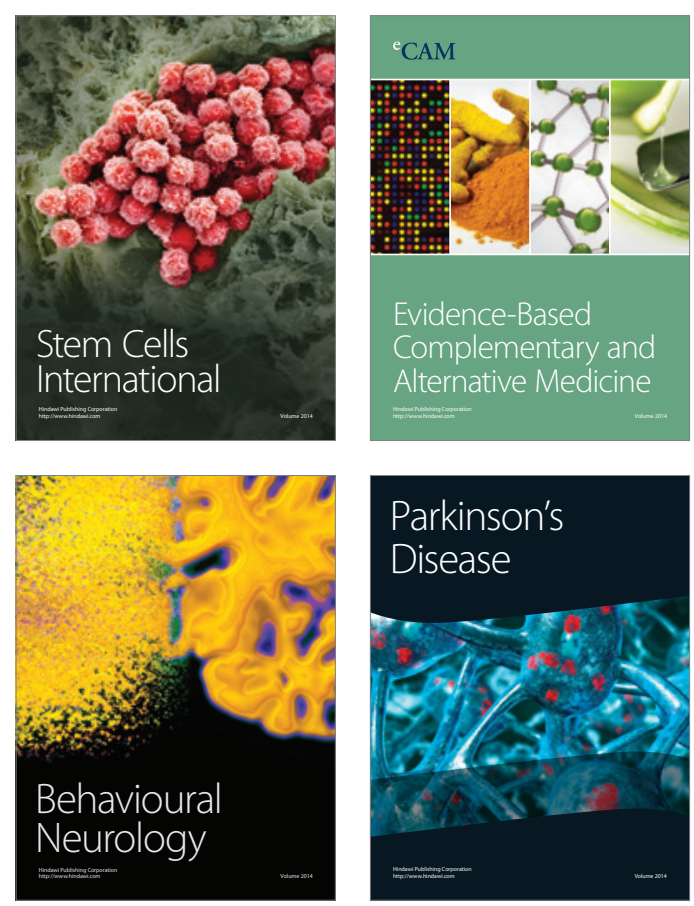

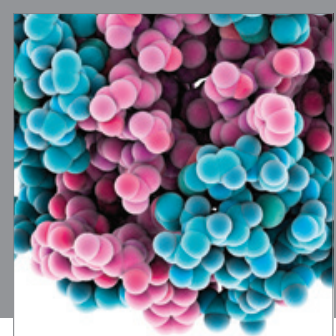

Journal of
Diabetes Research

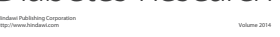

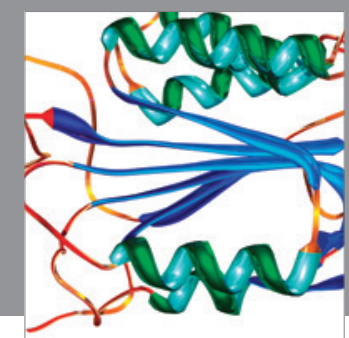

Disease Markers
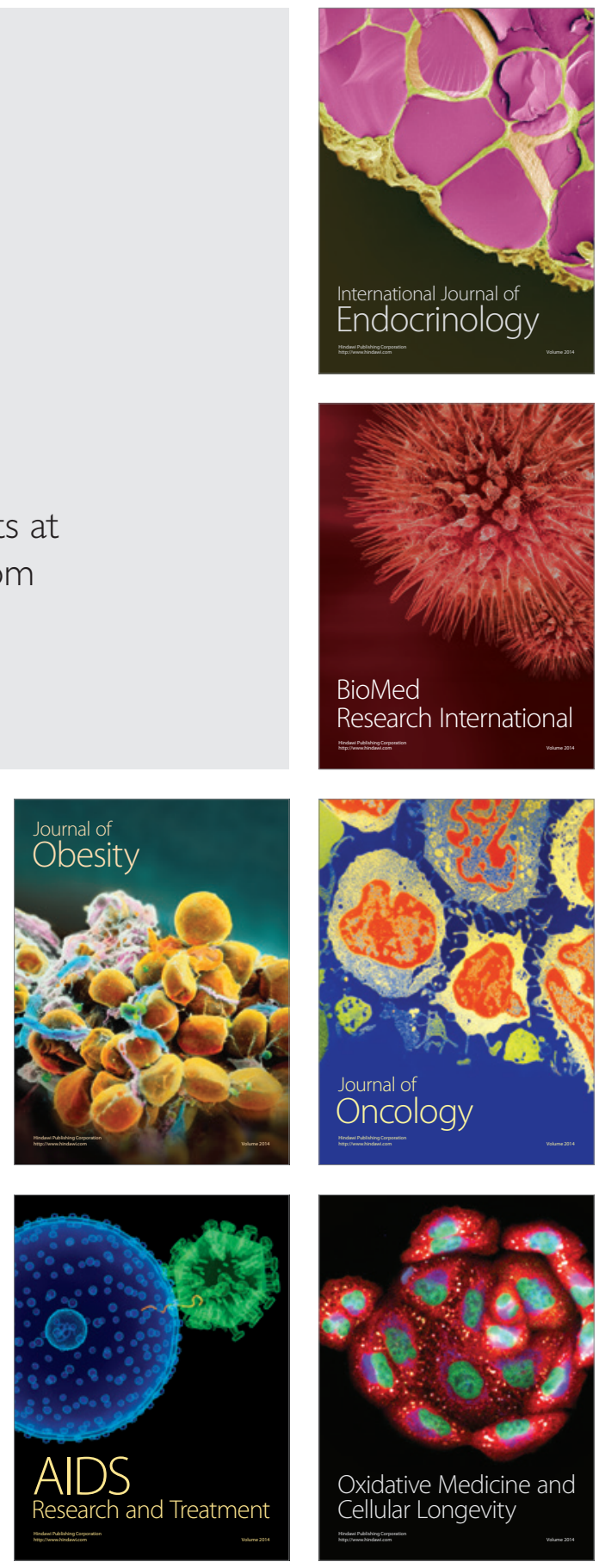